\title{
Efficacy of Ganoderma lucidum on plasma lipids and lipoproteins in rats fed with high cholesterol diet
}

\begin{abstract}
Purpose - The purpose of this paper is to report on the anti-cholesterol activities of Ganoderma lucidum on Sprague-Dawley white rats. Design/methodology/approach - Rats were divided into four groups with a sample size of six rats per groups. Different formula diets were given to each group for a period of six months. At regular intervals, blood samples were taken and analysed for the total cholesterol (TC), triglycerides (TG), high density lipoprotein cholesterol (HDL-C) and low density lipoprotein (LDL-C) levels. Findings Administration of hyperlipidemia diet in rats fed with 1 per cent cholesterol diet caused an increase in the TC, TG, as well as LDL-C level as compared to the control. A decrease of 23.6 per cent in the HDL-C level was also noted. However, supplementation of the feed with G. lucidum ( 0.1 per cent) in rats decreased the TC, TG and LDL-C level significantly $(\mathrm{P}<0.05)$ while further increased the plasma concentration of HDL-C. In the case of rats fed with diet containing a mixture of 1 per cent cholesterol and 0.1 per cent of Ganoderma, the lipid profile showed much higher readings $(\mathrm{p}<0.05)$ compared to the chol group. Practical implications - The possible efficacy of G. lucidum in reducing the deposition of cholesterol in the wall of the blood vessels was indicated. Originality/value - This article provides useful information to health care providers and consumers.
\end{abstract}

Keyword: Diet, Alternative medicines, Malaysia 